\title{
Gaussian and Golden Wavelets: A Comparative Study and their Applications in Structural Health Monitoring
}

\author{
F. E. GOSSLER ${ }^{1 *}$, B. R. OLIVEIRA ${ }^{2}$, M. A. Q. DUARTE ${ }^{3}$, J. VIEIRA FILHO $^{4}$, \\ F. VILLARREAL ${ }^{5}$ and R. L. LAMBLÉM ${ }^{6}$
}

Received on December 10, 2018 / Accepted on December 06, 2020

\begin{abstract}
In this work, a comparative analysis between Gaussian and Golden wavelets is presented. These wavelets are generated by the derivative of specific base functions. In this case, the order of the derivative also indicates the number of vanishing moments of the wavelet. Although these wavelets have a similar waveform, they have several distinct characteristics in time and frequency domains. These distinctions are explored here in the scale space. In order to compare the results provided by these wavelets for a real signal, they are used in the decomposition of a signal inserted in the context of structural health monitoring.
\end{abstract}

Keywords: gaussian wavelets, golden wavelets, vanishing moments, structural health monitoring.

\section{INTRODUCTION}

Wavelet analysis is a powerfull mathematical tool for several areas of science and engineering. There are several problems in different areas that can be explored and solved using this theory. Defined in a simple way, wavelets are analysis functions that satisfy certain conditions, mostly used in the Wavelet Transform (WT) $[5,16,17]$.

\footnotetext{
*Corresponding author: Fabrício Ely Gossler - E-mail: fabricio.ely@unesp.br

1 Department of Electrical Engineering, UNESP - São Paulo State University, Av. Professor José Carlos Rossi, 1370, 15385-000, Ilha Solteira, SP, Brasil. _ E-mail: fabricio.ely@unesp.br https://orcid.org/0000-0001-5095-1363

2 Department of Electrical Engineering, UNESP - São Paulo State University, Av. Professor José Carlos Rossi, 1370 15385-000, Ilha Solteira, SP, Brasil. - E-mail: bruno@editorapantanal.com.br https://orcid.org/0000-0002-1037-6541

3 Department of Mathematics, UEMS - Mato Grosso do Sul State University, Rodovia MS 306, km 6.4, 79540-000, Cassilândia, MS, Brasil. - E-mail: marco@uems.br https://orcid.org/0000-0003-2494-448X

4 Department of Electrical Engineering, UNESP - São Paulo State University, Av. Professor José Carlos Rossi, 1370 15385-000, Ilha Solteira, SP, Brasil. _ E-mail: jozue.vieira@unesp.br https://orcid.org/0000-0003-1274-5424

5 Department of Mathematics, UNESP - São Paulo State University, Alameda Rio de Janeiro, 266, 15385-000, Ilha Solteira, SP, Brasil. - E-mail: francisco.villarreal@unesp.br https://orcid.org/0000-001-8218-8593

6 Department of Mathematics, UEMS - Mato Grosso do Sul State University, Rodovia MS 306, km 6.4, 79540-000, Cassilândia, MS, Brasil. - E-mail: lamblem@uems.br https://orcid.org/ 0000-0002-6354-7375
} 
WT is a mathematical tool that splits signals into different frequency bands. As described by Daubechies [5], WT can be divided into two main types: Continuous Wavelet Transform (CWT) and the Discrete Wavelet Transform (DWT). The WT type choice depends on the objective and the problem to be analyzed. CWT is more inidicated for a detailed time-frequency (scale) analysis or precise localization of signal transients. Generally, DWT is used to obtain the sparsest possible signal representation for compression, denoising, or signal transmission [21]. In a general sense, this paper focuses on CWT.

The result of the WT depends directly on the wavelet function used. Thus, the wavelet choice must be made carefully, and it is done directly by the characteristics of the signal to be analyzed [17]. Wavelets can be complex or real-valued functions. Real wavelets are used to detect sharp signal transitions. On the other hand, analytic complex wavelets are used to identify instantaneous frequency evolution [16].

There exist many different types of wavelet functions to be chosen. Some wavelets belong to specific families such as the well-known Daubechies wavelets [5]. The derivatives of the Gaussian probability density function form another example of real wavelets family, known as Gaussian wavelets [24]. Another example recently presented is the Golden wavelets family, where the members are obtained by the derivatives of the quotient between two distinct Fibonaccicoefficient polynomials (FCPs) [10]. For both wavelet families, the order of the derivative also indicates the number of vanishing moments that the generated wavelet has. The number of vanishing moments is a very important property, since this value is directly related to the smoothness of the wavelet.

In Gossler et al. [11], a comparative analysis between Mexican and Golden Hat wavelets was presented. Mexican Hat is a well-known wavelet in the literature, defined as the second derivative of the Gaussian probability density function [5], and therefore, it is a Gaussian family member. Golden Hat wavelet is a Golden family member and it is generated by the fourth quocient derivative between two distinct FCPs. It was verified that, although Mexican and Golden Hat wavelets have similar waveforms, they can present significantly different results. In fact, a crucial difference between these wavelets is that Golden Hat has twice as much vanishing moments than Mexican Hat [11].

In this work, a comparative study will be presented considering a variety of Gaussian and Golden wavelets. Using the methodology similar to that presented in [11], some evaluation metrics will be used in order to characterize these wavelets in time and frequency domains. For a comparative analysis, twenty wavelet functions will be analyzed, ten of them belong to the Gaussian family, and the other ten are Golden wavelets. After comparing the wavelets using the chosen metrics, they will be used to decompose signals in the context of structural health monitoring (SHM) systems. The purpose of this application is to compare in practice the results obtained with the considered wavelets.

SHM based on wavelet analysis is a significant subject of research [26]. Taha et al. [22] presents a state-of-the-art review study of WT applied to SHM systems, where specific needs of SHM are 
addressed to WT. In the specialized literature, there is a variety of articles in which wavelets are proposed as a basic signal processing tool in SHM applications [8, 13, 14, 19, 20, 23].

The remainder of the paper is organized as follows. Firstly, a basic description of real wavelets and CWT from a signal analysis point of view is presented, as well as some of its mathematical properties and requirements. Then a comparative analysis between Gaussian and Golden wavelets is presented in qualitative and quantitative terms. It is followed by the application of these wavelets in the decomposition of real signals inserted in the SHM context. Finally, conclusions are drawn.

\section{REAL WAVELETS AND CONTINUOUS WAVELET TRANSFORM}

Wavelet analysis is often capable of revealing characteristics or data aspects of a signal, such as trends, breakdown points, discontinuities in higher derivatives, and self-similarity [5]. As well as Fourier analysis, wavelet analysis deals with expansions of basic functions. In signal processing, wavelet analysis can be used for a wide variety of fundamental tasks, being very useful in various application fields, e.g., seismology, turbulence analysis, computer graphics, astronomy, pattern recognition, quantum optics and biomedical engineering [16].

A family of functions of the form

$$
\psi_{s, \tau}(t)=\frac{1}{\sqrt{s}} \psi\left(\frac{t-\tau}{s}\right), s, \tau \in \mathbb{R}, s>0,
$$

generated from single function $\psi$ by the operations of dilations $s$ and translations $\tau$, such that

$$
\|\psi\|^{2}=\int_{-\infty}^{+\infty}|\psi(t)|^{2} d t<+\infty
$$

has been used frequently in recent years in various applications. These functions are called wavelets [12]. A real wavelet $\psi(t)$ satisfies the admissibility condition given by $[3,12]$ :

$$
c_{\psi}=\int_{0}^{+\infty} \frac{|\Psi(\Omega)|^{2}}{\Omega} d \Omega<+\infty,
$$

where

$$
\Psi(\Omega)=\int_{-\infty}^{+\infty} \psi(t) e^{-j \Omega t} d t
$$

is the Fourier Transform (FT) of $\psi(t), \Omega$ is the angular frequency parameter, and $j=\sqrt{-1}$ is the imaginary unit. $\psi(t)$ function is called a mother wavelet [17]. Condition (2.2) implies that $\int_{-\infty}^{+\infty} \psi(t) d t=\Psi(0)=0$, which means that wavelets are zero average functions [5].

For scaling $s(s>0)$ and translation $\tau$ parameters, and for a specific real wavelet choice $\psi(t)$, the CWT for a continuous time finite energy signal $x(t)$ is given by Eq. $(2.3)[5,16,17]$ :

$$
W_{x}^{\psi}(s, \tau)=\int_{-\infty}^{+\infty} x(t) \psi_{s, \tau}(t) d t
$$


Note that $W_{x}^{\psi}(s, \tau)$ measures, in a certain sense, the fluctuations of the signal $x(t)$ around the point $\tau$, at the scale given by $s$ [17]. When $s$ increases, $\psi(t)$ is expanded, and its frequency content moves to the lower frequency bands. Decreasing $s$ implies the compression of $\psi(t)$, and its frequency content moves to the higher bands. Generally, wavelet functions are considered to have unit energy, i.e., $\|\psi\|^{2}=1$, and the normalization factor $1 / \sqrt{s}$ ensures $\|\psi\|^{2}=\left\|\psi_{s, \tau}\right\|^{2}$.

Eq. (2.3) can also be rewritten as a convolutional product [16]. Therefore, the real CWT can be also interpreted as a frequency-based filtering of the signal by rewriting (2.3) as an Inverse Fourier Transform (IFT):

$$
W_{x}^{\psi}(s, \tau)=\frac{1}{2 \pi} \int_{-\infty}^{+\infty} X(\Omega) \sqrt{s} \Psi(s \Omega) e^{j \Omega \tau} d \Omega,
$$

where $X(\Omega)$ is the FT of the signal $x(t)$.

The information concerning the frequency content of the signal is provided by a relative frequency $f_{s}$ estimated from the mother wavelet center frequency $f_{c}$ in $\mathrm{Hz}$, the signal sampling rate $T_{s}$ and the decomposition scale $s$. To relate scale and frequency the following relation is used [1]:

$$
f_{s}=\frac{f_{c}}{T_{s} \times s}
$$

where $f_{c}$ can be estimated by association with a periodic signal or by the predominant frequency of $|\Psi(\Omega)|$. In [16], the expression given by Eq. (2.6) is presented to calculate the center angular frequency denoted by $\eta$. This parameter is associated with analytic complex wavelets, since $\Psi(\Omega)$ is zero at negative frequencies, i.e., $\Psi(\Omega)=0$ if $\Omega<0$.

$$
\eta=\frac{1}{2 \pi} \int_{0}^{+\infty} \Omega|\Psi(\Omega)|^{2} d \Omega
$$

The wavelet time location is defined by $\mu=\int_{-\infty}^{+\infty} t|\psi(t)|^{2} d t$. The spread around $\mu$ and $\eta$ are measured by the variances

$$
\sigma_{t}^{2}=\int_{-\infty}^{+\infty}|t-\mu|^{2}|\psi(t)|^{2} d t, \text { and } \sigma_{\Omega}^{2}=\frac{1}{2 \pi} \int_{0}^{+\infty}(\Omega-\eta)^{2}|\Psi(\Omega)|^{2} d \Omega
$$

respectively [16]. From the Heisenberg uncertainty principle, we obtain that $\sigma_{t} \times \sigma_{\Omega} \geq 1 / 2$, i.e., there is a minimum surface that limits the time-frequency resolution [7].

An important property of wavelets is the number of vanishing moments. The greater it is the smoother is the wavelet. Vanishing moments are crucial to measure the local regularity of a signal [16]. A wavelet $\psi(t)$ has $N$ vanishing moments if the following condition is verified [17]:

$$
\int_{-\infty}^{+\infty} t^{k} \psi(t) d t=0, k=0,1, \cdots, N-1,
$$

from which follows that any wavelet has at least one vanishing moment. Based on the vanishing moments definition (2.8) the following theorem can be proved [16]: 
Theorem 2.1 (). A wavelet $\psi(t)$ with a fast decay has $N$ vanishing moments if and only if there exists $\theta(t)$ with a fast decay such that

$$
\psi(t)=(-1)^{N} \frac{d^{N}}{d t^{N}} \theta(t)
$$

As a consequence

$$
W_{x}^{\psi}(s, \tau)=s^{N} \frac{d^{N}}{d \tau^{N}}\left(x \star \bar{\theta}_{s}\right)(\tau)
$$

with

$$
\bar{\theta}_{s}(t)=s^{-1 / 2} \theta(-t / s)
$$

Moreover, $\psi(t)$ has no more than $N$ vanishing moments if and only if

$$
\int_{-\infty}^{\infty} \theta(t) d t=K, K \neq 0
$$

The convolution $x \star \bar{\theta}_{s}$ can be interpreted as a weighted average of $x$ with a kernel dilated by $s$. In [16], Mallat also proved that the following equation holds:

$$
\lim _{s \rightarrow 0} \frac{W_{x}^{\psi}(s, \tau)}{s^{N+1 / 2}}=K \frac{d^{N}}{d \tau^{N}} x(\tau) .
$$

for an $N$ times continuously differentiable function $x(t)$ in the neighborhood of $\tau$.

From Eqs. (2.3), (2.4) and (2.10), it is clear that the coefficients $W_{x}^{\psi}(s, \tau)$ are the result from scale and translation parameters variations and the choice of the wavelet function, since these parameters are applied in order to change the function shape. In [24], Torrence and Compo list four determinant factors for this function choice, related to orthogonality, counter-domain, width and shape.

Orthogonal wavelets provide a fast implementation analogous to a filter bank approach, and they are generally used in DWT. On the other hand, for analysis purposes, the non-orthogonal wavelet functions considered in CWT are more suitable because their redundancy reveals spectral information of the signal. In time series analysis this non-orthogonal transform is useful since smooth and continuous fluctuations are expected [24].

Regardless of the wavelet function chosen, there exists a trade-off between its support (width) in time and frequency domains, which affects CWT resolution. If the wavelet function is narrow in time, then it has good time-resolution but poor frequency-resolution, and vice-versa, due to Heisenberg uncertainty principle. Meyer wavelets, for example, have finite support in frequency but infinite support in time [16]. Wavelet functions described in Section 3 has infinity ${ }^{1}$ support in time. However, there are examples of compactly supported wavelets, such as Daubechies orthogonal wavelets [5]. Thus, the choice of the wavelet function must prioritize the domain (time or frequency) that is expected to have a better resolution.

\footnotetext{
${ }^{1}$ Despite the infinite support, in practice it is considered an effective support, which is finite.
} 
The shapes of wavelet functions depend on the number of vanishing moments and on their symmetry with respect to the $y$-axis. In relation to symmetry, symmetric wavelets imply in linear phase response. This property is desirable in many problems due to waveform-preserving in time-domain. With the exception to the Haar wavelet, no other orthogonal compactly supported wavelet is symmetric [5]. Therefore, the wavelet choice is very important and depends on the signal features. Some wavelets belong to specific families generated by Eq. (2.9). Gaussian and Golden wavelets are examples of families generated from (2.9). Such wavelets are symmetric/antisymmetric, non-orthogonal and have infinite support in time domain. In the next Section these wavelet families are presented.

\section{GAUSSIAN AND GOLDEN WAVELETS}

By Theorem 2.1, it can be verified that for a particular choice of $\theta(t)$, wavelet functions can be obtained with a desired number of vanishing moments. An example of a function that can be used to obtain wavelets according to (2.9) is the Gaussian probability density function [24], defined by Eq. (3.1).

$$
\theta_{\text {gaus }}(t)=e^{-t^{2} / 2}
$$

Therefore, deriving the Gaussian probability density function, several wavelets can be obtained. Functions of the type

$$
\psi(t)=\frac{d^{p}}{d t^{p}} \theta_{\text {gaus }}(t)
$$

are known as Gaussian wavelets or derivative of a Gaussian (DOG) [24]. An example of a Gaussian wavelet is the Mexican Hat, obtained when $\mathrm{p}=2$ [5].

Golden wavelets are obtained by the $N$ th derivative of the quotient between two distinct FCPs [10]. A FCP of degree $n$ is defined by

$$
p_{n}(t)=\sum_{k=0}^{n} F_{k+1} t^{n-k},
$$

where $F_{k}=F_{k-1}+F_{k-2}, k \geq 2$, by setting $p_{0}(t)=1$ and $F_{0}=F_{1}=1$. The polynomial sequence $\left\{p_{n}(t)\right\}_{n=0}^{\infty}$ is called Fibonacci-coefficient polynomial sequence [9]. The Golden Hat wavelet is a Golden wavelet example, and it is defined as the fourth derivative of the function [11]

$$
\theta_{\text {gold }}(t)=\frac{1}{t^{2}+t+2}
$$

According to Eq. (2.9), the $N$ th derivatives of both functions defined in (3.1) and (3.2) result in wavelets with $N$ vanishing moments. Thus, using the property of the FT derivative, the FT of the Gaussian and Golden wavelets with $N$ vanishing moments, can then be calculated by Eqs. (3.3) and (3.4), respectively:

$$
\begin{aligned}
& \Psi_{\text {gaus }}(\Omega)=A_{N}(j \times \Omega)^{N} \Theta_{\text {gaus }}(\Omega), \\
& \Psi_{\text {gold }}(\Omega)=B_{N}(j \times \Omega)^{N} \Theta_{\text {gold }}(\Omega),
\end{aligned}
$$


where $\Theta_{\text {gaus }}(\Omega)$ and $\Theta_{\text {gold }}(\Omega)$ are the respective FT of $\theta_{\text {gaus }}(t)$ and $\theta_{\text {gold }}(t)$. Parameters $A_{N}$ and $B_{N}$ are constants satisfying $\|\Psi(\Omega)\|^{2}=2 \pi$.

For the comparative analysis in this study, twenty wavelet functions will be analyzed, ten from Gaussian family, and ten from Golden family. In order to facilitate the notation, gaus $N$ and gold $N$ notations will be used, where $N$ indicates the number of vanishing moments and also the derivative order:

$$
\begin{gathered}
\operatorname{gaus} N=C_{N} \frac{d^{N}}{d t^{N}} \theta_{\text {gaus }}(t), N=1, \ldots, 10, \\
\operatorname{gold} N=D_{N} \frac{d^{N}}{d t^{N}} \theta_{\text {gold }}(t-1 / 2), N=1, \ldots, 10,
\end{gathered}
$$

where $C_{N}$ and $D_{N}$ are chosen in such a way to ensure unit energy. As it can be seen in Eq. (3.6), a delay of $1 / 2$ unit is applied in $\theta_{\text {gold }}(t)$ function. It is due to the fact that the Golden wavelets are located at $t=0$.

In Figure 1 gaus $N$ and gold $N$ waveforms are shown (with normalized amplitudes), for $N=1,5,8$. Qualitatively, for the same number of vanishing moments, it is remarkable that such wavelets have a certain similarity waveforms. Although, in [11] it was shown that gaus2 (Mexican hat) and gold4 (Golden Hat) have a high degree of similiarity, Golden wavelets have less expressive oscillations than Gaussian wavelets. The main difference comes from the support between them. As they are generated, Golden wavelets have an effective lower support than Gaussian wavelets.

In order to quantify the comparison between the wavelets, evaluation metrics will be computed for the purpose of characterizing differences between the functions in time and frequency domains. The chosen metrics are the variances $\sigma_{t}^{2}$ and $\sigma_{\Omega}^{2}$, the product $\sigma_{t} \times \sigma_{\Omega}$, the center frequency $\eta$ and the predominant frequency $\Omega_{p}$, the bandpass $\left[\Omega_{L B}, \Omega_{U B}\right]$, where $\Omega_{L B}$ and $\Omega_{U B}$ are respectively lower and upper frequency limits, and finally, the bandwidth $\Delta_{\Omega}=\left|\Omega_{U B}-\Omega_{L B}\right|$. Bandpass and bandwidth were characterized by the magnitude spectrum with normalized amplitude. The bandpass was calculated using a cutoff frequency of $6 \mathrm{~dB}$ attenuation (close to 0.5 relative to peak). In Table 1, the computed metrics are shown for each considered wavelet.

Observing Table 1, it can be seen the evolution of $\sigma_{t}^{2}$ and $\sigma_{\Omega}^{2}$. The $\sigma_{t}^{2}$ value tends to decrease when the number of vanishing moments increases, and, on the other hand, $\sigma_{\Omega}^{2}$ tends to increase when the number of vanishing moments increases. It means that, the greater the number of vanishing moments is, the better is the resolution in time domain, and it is most evident for Golden wavelets. Therefore, the time spread $\sigma_{t}^{2}$ for a specific wavelet gold $N$ is much smaller than for gaus $N$, except when $N=1$. This is expected, since the effective gold $N$ support is smaller than that of gaus $N$, as it can be seen in Figure 1.

It is noteworthy that $\sigma_{\Omega}^{2}$ values indicate that Gaussian wavelets have approximately the same frequency dispersion, independent of the number of vanishing moments. On the other hand, Golden wavelets have a frequency dispersion that varies significantly, where it is verified that the greater is the number of vanishing moments, the greater is $\sigma_{\Omega}^{2}$. Note that the time-frequency resolution is more accurate when $N$ also increases. Thus, the product $\sigma_{t} \times \sigma_{\Omega}$ also decreases as the number of vanishing moments increases. 

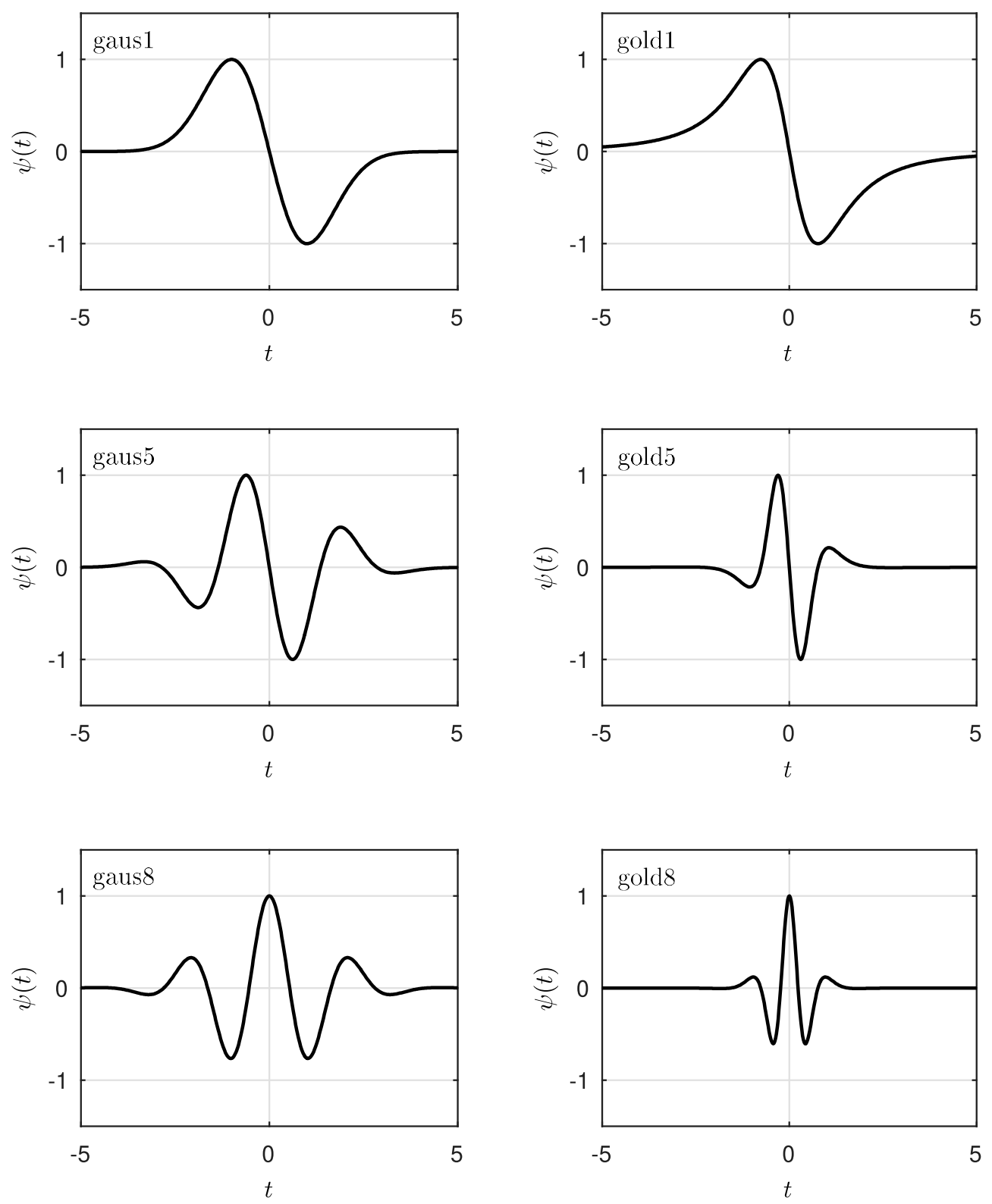

Figure 1: Gaussian and Golden wavelets.

Since the magnitude spectra of the wavelets are asymmetric, the center frequency is not the peak frequency for Gaussian and Golden wavelets. In relation to the bandpass and bandwidth, note that $\Delta_{\Omega}$ does not vary greatly for gaus $N$ wavelets, although bandpass has changed. For 
Table 1: Evaluation Metrics for Gaussian and Golden wavelets.

\begin{tabular}{cccccccc}
\hline Wavelet & $\sigma_{t}^{2}$ & $\sigma_{\Omega}^{2}$ & $\sigma_{t} \times \sigma_{\Omega}$ & $\eta$ & $\Omega_{p}$ & {$\left[\Omega_{L B}, \Omega_{U B}\right]$} & $\Delta_{\Omega}$ \\
\hline gaus1 & 1.50 & 0.22 & 0.57 & 1.12 & 1.00 & {$[0.31,1.92]$} & 1.61 \\
gaus2 & 1.16 & 0.24 & 0.50 & 1.50 & 1.41 & {$[0.68,2.31]$} & 1.63 \\
gaus3 & 1.10 & 0.24 & 0.51 & 1.80 & 1.73 & {$[0.97,2.62]$} & 1.65 \\
gaus4 & 1.07 & 0.24 & 0.50 & 2.06 & 2.00 & {$[1.23,2.88]$} & 1.65 \\
gaus5 & 1.05 & 0.24 & 0.50 & 2.29 & 2.23 & {$[1.46,3.11]$} & 1.65 \\
gaus6 & 1.04 & 0.25 & 0.50 & 2.50 & 2.44 & {$[1.67,3.32]$} & 1.65 \\
gaus7 & 1.03 & 0.25 & 0.50 & 2.69 & 2.64 & {$[1.86,3.51]$} & 1.65 \\
gaus8 & 1.03 & 0.25 & 0.50 & 2.87 & 2.82 & {$[2.04,3.69]$} & 1.65 \\
gaus9 & 1.02 & 0.25 & 0.50 & 3.04 & 3.00 & {$[2.21,3.86]$} & 1.65 \\
gaus10 & 1.02 & 0.25 & 0.50 & 3.20 & 3.16 & {$[2.37,4.02]$} & 1.65 \\
\hline gold1 & 1.75 & 0.42 & 0.85 & 1.13 & 0.75 & {$[0.17,2.02]$} & 1.85 \\
gold2 & 0.58 & 0.71 & 0.64 & 1.88 & 1.51 & {$[0.57,3.14]$} & 2.57 \\
gold3 & 0.35 & 1.00 & 0.59 & 2.64 & 2.26 & {$[1.05,4.17]$} & 3.12 \\
gold4 & 0.25 & 1.28 & 0.56 & 3.40 & 3.02 & {$[1.57,5.17]$} & 3.60 \\
gold5 & 0.19 & 1.57 & 0.54 & 4.15 & 3.77 & {$[2.12,6.13]$} & 4.01 \\
gold6 & 0.15 & 1.85 & 0.52 & 4.91 & 4.53 & {$[2.69,7.07]$} & 4.38 \\
gold7 & 0.13 & 2.14 & 0.52 & 5.66 & 5.29 & {$[3.27,8.01]$} & 4.74 \\
gold8 & 0.11 & 2.42 & 0.51 & 6.42 & 6.04 & {$[3.86,8.92]$} & 5.06 \\
gold9 & 0.10 & 2.71 & 0.52 & 7.18 & 6.80 & {$[4.47,9.83]$} & 5.36 \\
gold10 & 0.09 & 3.00 & 0.51 & 7.93 & 7.55 & {$[5.08,10.74]$} & 5.66 \\
\hline & & & & & & &
\end{tabular}

Golden wavelets, $\Delta_{\Omega}$ varies significantly according to the number of vanishing moments. It can be explained by the frequency dispersion $\sigma_{\Omega}^{2}$ value.

\section{GAUSSIAN AND GOLDEN WAVELETS APPLICATION}

In order to show the different results obtained from Gaussian and Golden wavelets, in this Section they will be used in the decomposition of real signals. The signals used for such analyzes represent electrical voltages for a given sensor in the context of SHM systems based on the electromechanical impedance (EMI) principle.

\subsection{Structural Health Monitoring - SHM}

SHM aims to monitor the integrity of a structure in order to minimize repair and maintenance costs. In this way, such systems can provide a greater level of security to users, avoiding possible catastrophic failures. Therefore, SHM is an important research subject with many applications in different areas, such as mechanical, aerospace and civil engineering [6]. 
The main techniques used in SHM systems have their origin in nondestructive evaluation (NDE) methods. In this context, the method based on EMI principle has been considered as one of the most promising $[4,18,25]$. Such method was initially proposed by Liang and Sun [15], and basically consists in monitoring the mechanical impedance in a structure caused by a damage presence.

Typical SHM applications generally use commercial impedance analyzer with a high cost or impedance measurement systems based on frequency response functions (FRF). The EMI-based SHM technique also stands out for its simplicity and the use of PZT (Lead Zirconate Titanate) piezoelectric transducers that are low cost components [25]. In such systems, to detect and locate damages in a structure, PZT piezoelectric actuators/sensors are used to excite/measure electrical impedance at high frequency ranges. In this case, damage identification is made by comparing the electrical impedance of the transducer measured with the structure in an initial condition, considered integral, with the impedance measured after the structure has suffered a possible damage. Generally, this comparison is performed by means of metric failure indexes, such as Correlation Coefficient Deviation Metric (CCDM) defined by Eq. (4.1).

In [2] and [25], methods for measuring electrical impedance in frequency and time domains, respectively, were presented. In [25], it was shown that PZT time response is enough to get any variation in the structure condition, and therefore, it is not necessary to perform FT to obtain the FRF.

\subsection{General description}

The goal of this case study is to analyze the time-domain response of a PZT sensor using Gaussian and Golden wavelets. Thus, the results obtained by these wavelets can express, in practice, the differences between them. The analyzed signals were provided by the Laboratory of Signal Processing and Instrumentation (LabPSI) from São Paulo State University - Ilha Solteira campus. Such signals were experimentally obtained and were used in [4], where an SHM system is based on the one proposed in [25]. Signals were acquired using a sensor/actuator, a piezoelectric capsule, model 7BB-27-4 from Murata, adhered to a $500 \mathrm{~mm}$ x $30 \mathrm{~mm}$ x $2 \mathrm{~mm}$ aluminum bar.

In [4], four damages were simulated in different locations, for distances at $10 \mathrm{~cm}, 20 \mathrm{~cm}, 30 \mathrm{~cm}$ and $40 \mathrm{~cm}$ from the sensor. All measurements were made with the structure in the free-free configuration, i.e., with the two extrema suspended by elastic and ambient temperature.

In this work, the scenario of experiments is the same as in [4], where the chirp excitation signal was adhered with an initial and final frequency of $3 \mathrm{kHz}$ and $35.6 \mathrm{kHz}$, respectively, and with a sampled rate at $250 \mathrm{kHz}$. In this way, five different signals are analyzed. One of these signals, denoted $V_{B}(t)$, represents the time response of the pair PZT-Structure in healthy condition. The other signals, denoted $V_{D 1}(t), V_{D 2}(t), V_{D 3}(t)$ and $V_{D 4}(t)$, represent the structure with simulated damages at $10 \mathrm{~cm}, 20 \mathrm{~cm}, 30 \mathrm{~cm}$ and $40 \mathrm{~cm}$ distance from the sensor, respectively. Figure 2 presents time responses for $V_{B}(t)$ and $V_{D 1}(t)$. 
(a)

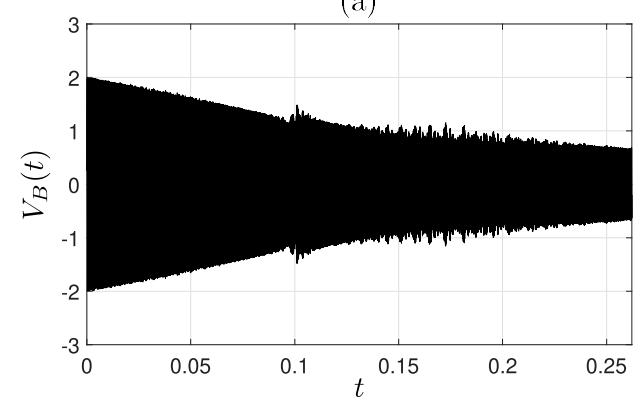

(b)

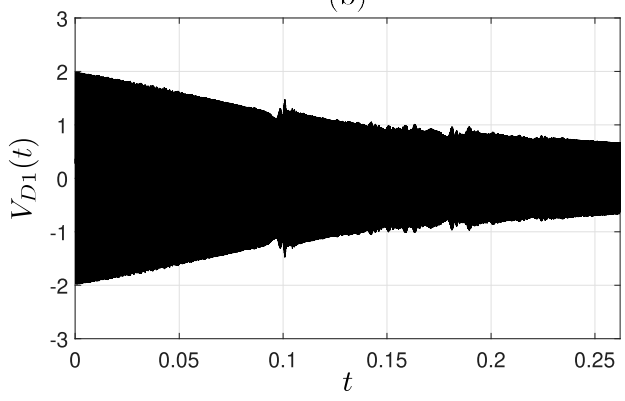

Figure 2: Signals (a) $V_{B}(t)$ and (b) $V_{D 1}(t)$.

\subsection{Methodology}

The application will follow the script: CWT will be applied to the signal $V_{B}(t)$ (also known as baseline) and to the signals $V_{D 1}(t), V_{D 2}(t), V_{D 3}(t)$ and $V_{D 4}(t)$. CWT will be applied in a certain range of scales. This range will be determined according to the wavelet and the spectral content of the analyzed signal. Therefore, the initial scale $s_{i}$ corresponds to the maximum relative frequency contained in the signals $(35.6 \mathrm{kHz})$, and the final scale $s_{f}$ corresponds to the lowest relative frequency $(3 \mathrm{kHz})$. In this case, to determine the range of scales for the CWT computation, the relation expressed by (2.5) will be used:

$$
s_{i}=\frac{f_{c}}{T_{s} \times f_{\max }}, s_{f}=\frac{f_{c}}{T_{s} \times f_{\min }},
$$

where $f_{\min }$ and $f_{\max }$ correspond to the minimum and the maximum frequency contained in the signal, respectively.

After determining the scale range $\left[s_{i}, s_{f}\right]$ corresponding to each wavelet, CWT is then applied to the signals $V_{B}(t)$ and $V_{D}(t)$. In practice, CWT is computed using $\psi(t)$ discretized version and parameters $s$ and $\tau$. In this case, we use the notation $W_{x}^{\psi}[s, \tau]$ to denote the discretized wavelet coefficients. It is expected that there is a difference between the CWT coefficients $W_{V_{B}}^{\psi}[s, \tau]$ and $W_{V_{D}}^{\psi}[s, \tau]$. To quantify this difference, CCDM will be computed:

$$
\mathrm{CCDM}=1-\left|\frac{\sum_{\tau}\left(W_{V_{B}}^{\psi}[s, \tau]-\overline{W_{V_{B}}^{\psi}}\right)\left(W_{V_{D}}^{\psi}[s, \tau]-\overline{W_{V_{D}}^{\psi}}\right)}{\sqrt{\sum_{\tau}\left(W_{V_{B}}^{\psi}[s, \tau]-\overline{W_{V_{B}}^{\psi}}\right)^{2}} \sqrt{\sum_{\tau}\left(W_{V_{D}}^{\psi}[s, \tau]-\overline{W_{V_{D}}^{\psi}}\right)^{2}}}\right|,
$$

where the variables $\overline{W_{V_{B}}^{\psi}}$ and $\overline{W_{V_{D}}^{\psi}}$ are the averaged values of $W_{V_{B}}^{\psi}[s, \tau]$ and $W_{V_{D}}^{\psi}[s, \tau]$, respectively. The value obtained by Eq. (4.1) will varies between 0 and 1. As it is described in [26], if $\mathrm{CCDM} \longrightarrow 0$ there is no damage, and if it increases towards unity, there is a high probability of damage. However, the damage can be easily detected with small values of CCDM [26].

In order to compare the results obtained in the wavelet domain, CCDM in time domain will be also computed. Table 2 shows CCDM results obtained in time domain for each considered 
damage. Such values will serve as reference for comparisons with CCDM values obtained in the wavelet domain.

Table 2: CCDM of the signals in the time domain

\begin{tabular}{ccccc}
\hline & Damage $10 \mathrm{~cm}$ & Damage $20 \mathrm{~cm}$ & Damage $30 \mathrm{~cm}$ & Damage $40 \mathrm{~cm}$ \\
\hline CCDM & 0.001789 & 0.001940 & 0.001977 & 0.001593 \\
\hline
\end{tabular}

\subsection{Results and Discussions}

Applying CWT to the signals, and then calculating the CCDM among CWT coefficients from integrated and damaged structure, greater values than those presented in Table 2 can be obtained. For each considered wavelet, Table 3 shows the normalized maximum CCDM calculated in the wavelet domain. These values are normalized in relation to CCDM values shown in Table 2:

$$
\mathrm{CCDM}_{w / t}=\mathrm{CCDM}_{w} / \mathrm{CCDM}_{t}
$$

where $\mathrm{CCDM}_{w}$ and $\mathrm{CCDM}_{t}$ are the respective CCDM computed in wavelet and time domains, respectively. Therefore, $\mathrm{CCDM}_{w / t}$ also indicates how efficient the difference found in the wavelet domain was in relation to that computed in time domain.

As it can be observed, CCDM values are higher for wavelets with more vanishing moments. This behavior was observed for both wavelet families considered. From Figure 1, it is possible to note that wavelets with larger number of vanishing moments have more considerable oscillations. Therefore, the expressive variations between the signals can be more efficiently detected with wavelets containing more vanishing moments, since CWT measures the similiarity between a signal and the wavelet used. For a wavelet with $N$ vanishing moments, gaus $N$ implies in a higher CCDM than gold $N$. It can be explained by the fact that the Gaussians have a better time-frequency resolution and more expressive oscillations than Golden wavelets.

In Figure 3-(a) the evolutions of CCDM indices for gaus2 (solid line) and gold2 (dashed line) are shown for each considered scale. These values refer to signals $V_{B}(t)$ and $V_{D 1}(t)$. It was observed that the CCDM characteristic has the same evolution behavior for the other wavelets, i.e., for a given range of scales, a distributive behavior is observed such that CCDM $>1$ from normalized results. This range is of interest, since it presents a greater index than the reference CCDM obtained in time domain. Figures 3-(b) and (c), CWT coefficients (at scale $s=2$ ) from gaus2 and gold 2 are shown for just one stretch of the signal. Note the difference between the damage and undamage structures signals.

As discussed earlier, Golden wavelets have a better time resolution than Gaussian (for $N>1$ vanishing moments). It is expected that the detection and location of transients and singularities present in the signals are different for both wavelets. Figures 4 and 5 show the CWT coefficients at scales $s=1$ and $s=3$, respectively, in relation to a simulated $V_{B}(t)$ singularity at $t=0.2324$. 
Table 3: Normalized maximum CCDM at wavelet domain.

\begin{tabular}{ccccc}
\hline wavelet & Damage 10cm & Damage 20cm & Damage 30cm & Damage 40cm \\
\hline gaus1 & 1.922855 & 2.015290 & 2.086269 & 2.018898 \\
gaus2 & 2.405184 & 2.591219 & 2.756180 & 2.638077 \\
gaus3 & 2.697404 & 2.940534 & 3.144309 & 2.959729 \\
gaus4 & 2.935967 & 3.230968 & 3.479868 & 3.226715 \\
gaus5 & 3.135846 & 3.484975 & 3.773217 & 3.458381 \\
gaus6 & 3.314585 & 3.711504 & 4.039214 & 3.660776 \\
gaus7 & 3.472759 & 3.914407 & 4.275399 & 3.843052 \\
gaus8 & 3.616134 & 4.097480 & 4.497113 & 4.005290 \\
gaus9 & 3.748165 & 4.265711 & 4.699647 & 4.152437 \\
gaus10 & 3.870505 & 4.421715 & 4.886158 & 4.286010 \\
\hline gold1 & 1.527296 & 1.554084 & 1.565667 & 1.536037 \\
gold2 & 2.086717 & 2.193278 & 2.269089 & 2.185383 \\
gold3 & 2.400714 & 2.578198 & 2.721787 & 2.596156 \\
gold4 & 2.566102 & 2.789125 & 2.987777 & 2.837845 \\
gold5 & 2.696406 & 2.946259 & 3.163545 & 2.984424 \\
gold6 & 2.814315 & 3.090860 & 3.327588 & 3.116043 \\
gold7 & 2.922626 & 3.225772 & 3.482269 & 3.238515 \\
gold8 & 3.022792 & 3.351048 & 3.626980 & 3.351615 \\
gold9 & 3.117898 & 3.470344 & 3.765215 & 3.459285 \\
gold10 & 3.205786 & 3.582656 & 3.897479 & 3.560578 \\
\hline
\end{tabular}

As it can be seen from Figures 4 and 5, the considered wavelets detected the signal oscillations at low scales. It can be observed that gaus 1 and gold1 do not detect the singularity efficiently for both scales. Furthermore, these wavelets provide very similar results. For $s=1$, CWT coefficients show that the singularity is efficiently detected by gaus5, gold5, gaus8 and gold8 wavelets. On the other hand, for $s=3$, the singularity is not detected by gaus 5 and gaus 8 . This is due to the fact that the detected oscillations of the signal overlap the singularity amplitude. It does not occur for gold5 and gold8 wavelets.

\section{CONCLUSIONS}

In this work comparisons between Gaussian and Golden wavelets families were performed, showing similarities and distinctions between them. Through this work it could be concluded that the Gaussian wavelets considered maintain approximately the same time and frequency resolutions, independent of the number of vanishing moments. On the other hand, the time and frequency resolutions for Golden wavelets change significantly as the number of vanishing moments varies. For Golden wavelets, the greater is the number of vanishing moments, the better is the resolution in time, and consequently, implying a poor resolution in the frequency domain. 
(a)

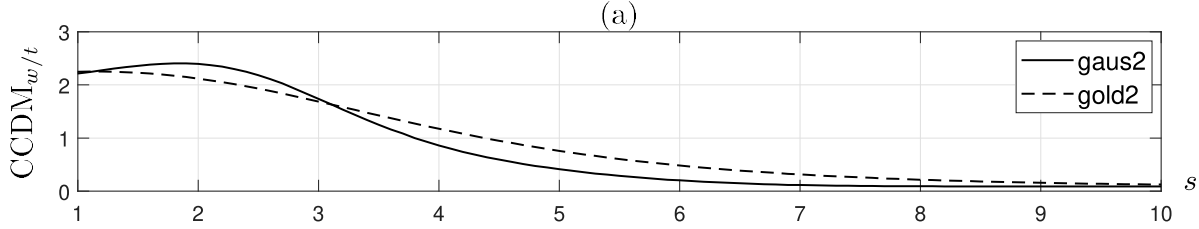

(b)

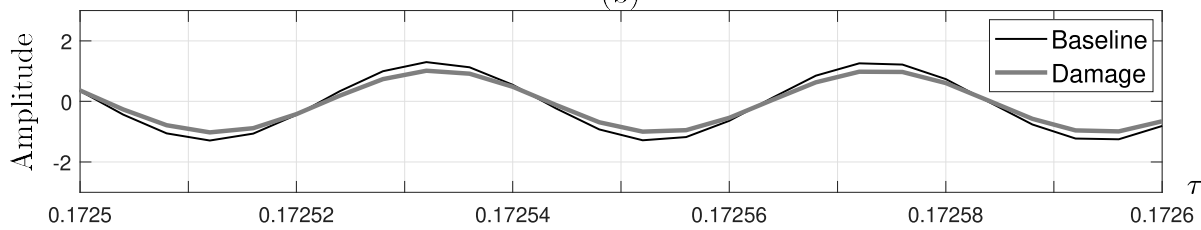

(c)

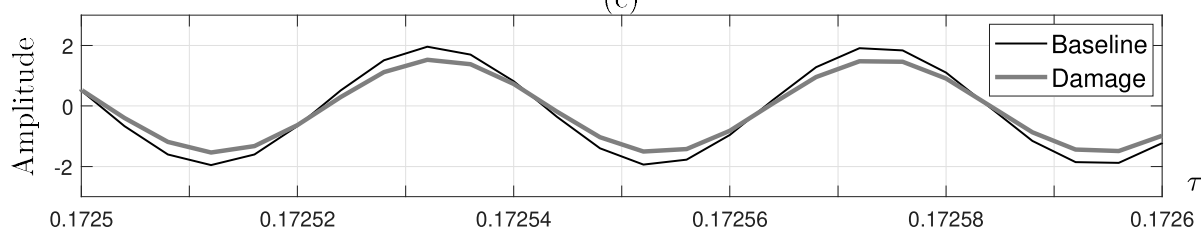

Figure 3: (a) CCDM index evolution using gaus2 (dashed line) and gold2 (solid line) wavelets, according to the scale. A stretch of CWT coeficients from $V_{B}$ (gray line) and $V_{D 1}$ (black line), using (b) gaus2 and (c) gold2 wavelets.
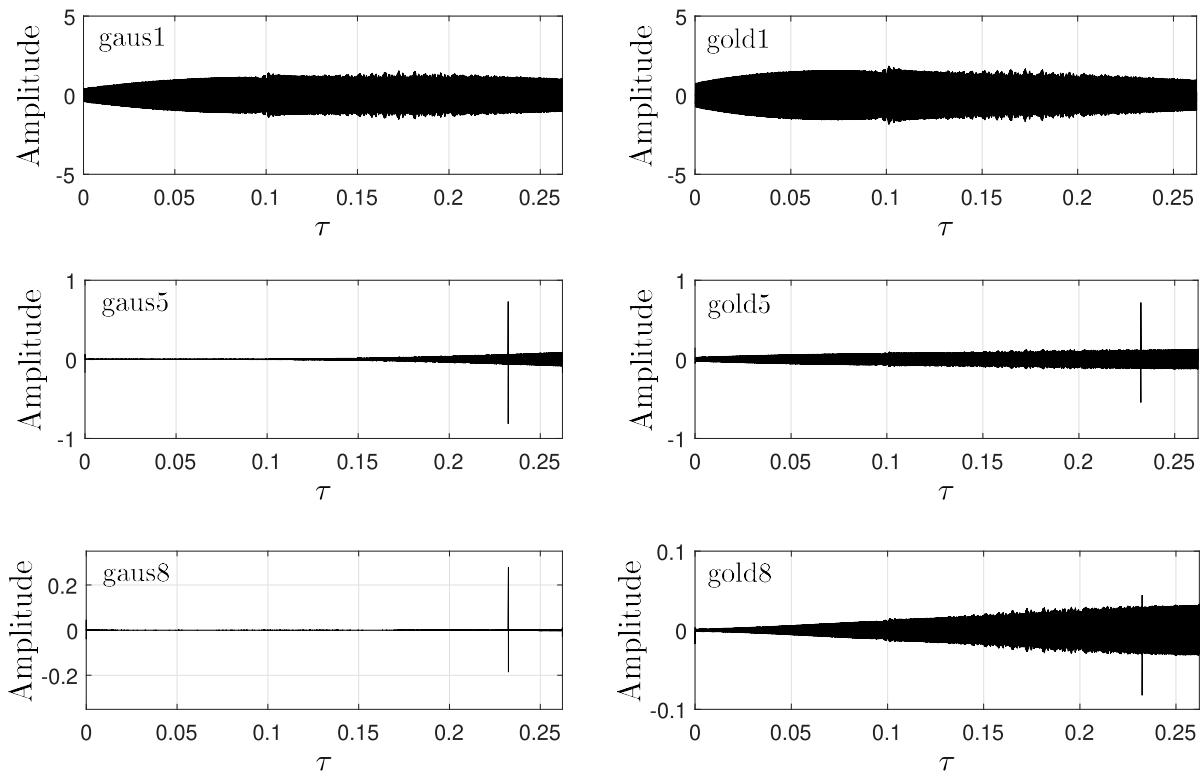

Figure 4: CWT coefficients at scale $s=1$ from signal $V_{B}(t)$ with a simulated singularity in $t=0.2324$. 

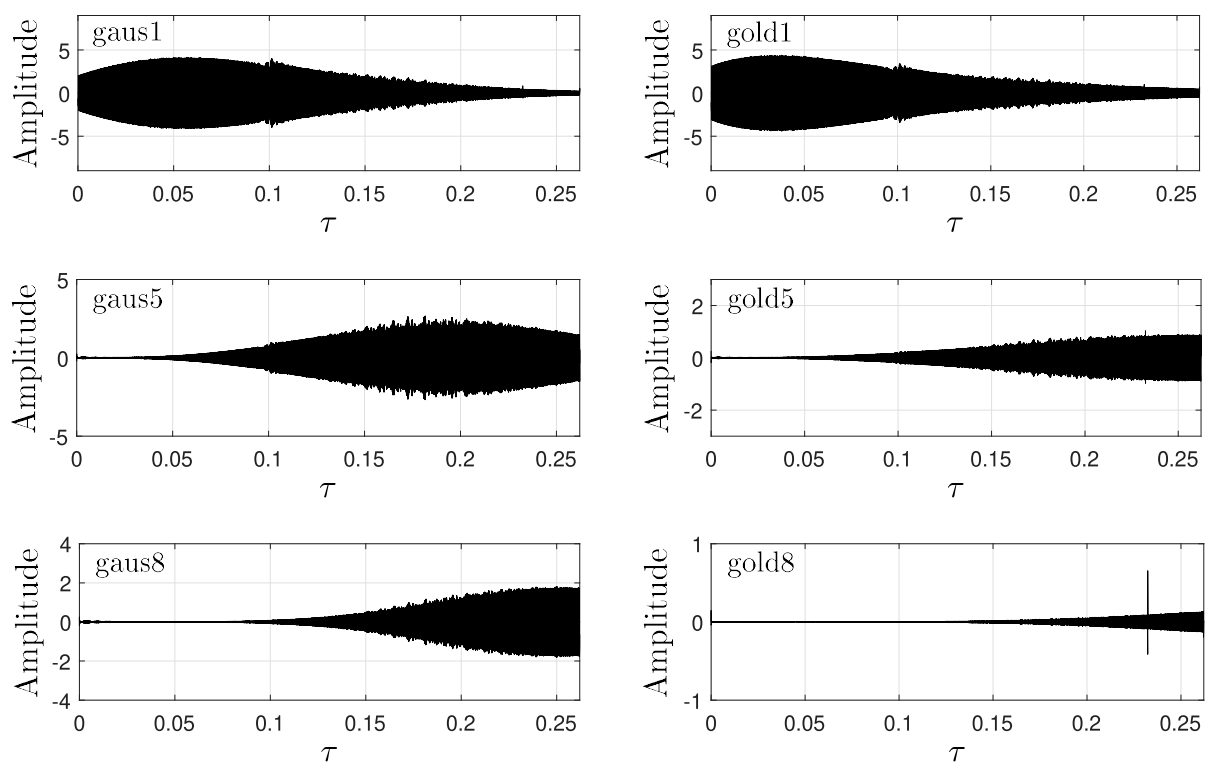

Figure 5: CWT coefficients at scale $s=3$ from signal $V_{B}(t)$ with a simulated singularity in $t=0.2324$.

Gaussian and Golden wavelets were used to decompose a signal in the context of SHM systems. The application shown in this paper indicates that the difference between the signals representing undamaged and damaged structures can be more expressive in the wavelet domain for a scale range. This could be verified by comparing CCDM in time and wavelet domains. It was verified that CCDM value is directly proportional to the number of vanishing moments in the used wavelet. For the considered application, Gaussians presented more expressive CCDM values than Golden wavelets.

Since Gaussian and Golden wavelets have very similar waveforms, the differences from the results obtained in the application show that not only the wavelet waveform should be taken into account, but also its time-frequency resolution. It is clear from the application shown in this paper, since the difference in results between gaus $N$ and gold $N$ is quite evident, although they have very similar waveforms. Preliminary results show that the detection of transients or singularities present in the signals can be performed more efficiently with Golden wavelets. It can also be explained by the fact that Golden wavelets have good resolution in time domain.

\section{Acknowledgements}

This work was supported by CAPES-Brazil (Coordination for the Improvement of Higher Education Personnel (CAPES) - Finance Code 001. 
RESUMO. Neste trabalho, uma análise comparativa entre as wavelets Gaussianas e Golden é apresentada. Tais wavelets são geradas pela derivada de uma função basica específica. Nesse caso, a ordem da derivada também indica o número de momentos nulos da wavelet. Embora essas wavelets tenham formas de onda muito semelhantes, elas possuem várias características distintas nos domínios do tempo e da frequência. Essas distinções são exploradas aqui no espaço de escalas. A fim de comparar os resultados das wavelets para um sinal real, essas wavelets são usadas na decomposição de um sinal inserido no contexto do sistemas de monitoramento de integridade estrutural.

Palavras-chave: wavelets gaussiana, wavelets golden, momentos nulos, monitoramento de integridade estrutural.

\section{REFERENCES}

[1] P. Abry. "Ondelettes et turbulences: multirésolutions, algorithmes de décomposition, invariance d'échelle et signaux de pression”. Diderot multimédia (1997).

[2] F.G. Baptista \& J. Vieira Filho. A new impedance measurement system for PZT-based structural health monitoring. IEEE Transactions on Instrumentation and Measurement, 58(10) (2009), 3602-3608.

[3] A.P. Calderon. Intermediate spaces and interpolation: the complex method. Studia Mathematica, 24(1) (1964), 113-190.

[4] N.E. Cortez Ledesma. "Development and implementation of a system for fault detection in structures using microcontroller (in Portuguese)". Master's thesis (2012).

[5] I. Daubechies. "Ten lectures on wavelets". Society for Industrial and Applied Mathematics (1992).

[6] C.R. Farrar \& K. Worden. "Structural health monitoring: a machine learning perspective". John Wiley and Sons (2013).

[7] D. Gabor. Theory of communication. Part 1: The analysis of information. Journal of the Institution of Electrical Engineers-Part III: Radio and Communication Engineering, 93(26) (1946), 429-441.

[8] A. Garofalo, N. Testoni, A. Marzani \& L. De Marchi. Wavelet-based Lamb waves direction of arrival estimation in passive monitoring techniques. In "Ultrasonics Symposium (IUS), 2016 IEEE International”. IEEE (2016), pp. 1-4.

[9] D. Garth, D. Mills \& P. Mitchell. Polynomials generated by the Fibonacci sequence. Journal of Integer Sequences, 10(2) (2007), 3.

[10] F.E. Gossler. "Wavelets and Fibonacci-coefficient polynomials (in Portuguese)". Master's thesis, Universidade Estadual Paulista (UNESP) - Ilha Solteira (2016).

[11] F.E. Gossler, B.R. Oliveira, J. Vieira Filho, F. Villarreal, M.A.Q. Duarte \& R.L. Lamblém. A Comparative Study between Mexican and Golden Hat Wavelets. Proceeding Series of the Brazilian Society of Computational and Applied Mathematics, (2018).

[12] A. Grossmann \& J. Morlet. Decomposition of Hardy functions into square integrable wavelets of constant shape. SIAM journal on mathematical analysis, 15(4) (1984), 723-736. 
[13] R. Janeliukstis, S. Rucevskis, P. Akishin \& A. Chate. Wavelet transform based damage detection in a plate structure. Procedia engineering, 161 (2016), 127-132.

[14] R. Janeliukstis, S. Rucevskis, M. Wesolowski \& A. Chate. Experimental structural damage localization in beam structure using spatial continuous wavelet transform and mode shape curvature methods. Measurement, 102 (2017), 253-270.

[15] C. Liang, F. Sun \& C. Rogers. Coupled electro-mechanical analysis of adaptive material systemsdetermination of the actuator power consumption and system energy transfer. Journal of intelligent material systems and structures, 8(4) (1997), 335-343.

[16] S. Mallat. "A wavelet tour of signal processing: the sparse way". Academic press (2008).

[17] Y. Meyer. "Wavelets: algorithms and applications". Society for Industrial and Applied Mathematics (1993).

[18] G. Park, H. Sohn, C. Farrar \& D. Inman. Overview of piezoelectric impedancebased health monitoring and path forward. The Shock and Vibration Digest, 35(6) (2003), 451-463.

[19] L. Ramesh \& P.S. Rao. Damage Detection in Structural Beams Using Model Strain Energy Method and Wavelet Transform Approach. Materials Today: Proceedings, 5(9) (2018), 19565-19575.

[20] M. Solís, M. Algaba \& P. Galvín. Continuous wavelet analysis of mode shapes differences for damage detection. Mechanical Systems and Signal Processing, 40(2) (2013), 645-666.

[21] G. Strang \& T. Nguyen. "Wavelets and filter banks". Society for Industrial and Applied Mathematics (1996).

[22] M.R. Taha, A. Noureldin, J. Lucero \& T. Baca. Wavelet transform for structural health monitoring: a compendium of uses and features. Structural Health Monitoring, 5(3) (2006), 267-295.

[23] A. Tjirkallis \& A. Kyprianou. Damage detection under varying environmental and operational conditions using Wavelet Transform Modulus Maxima decay lines similarity. Mechanical Systems and Signal Processing, 66 (2016), 282-297.

[24] C. Torrence \& G.P. Compo. A Practical Guide to Wavelet Analysis. Bulletin of the American Meteorological Society, 79(1) (1998).

[25] J. Vieira Filho, F.G. Baptista, J. Farmer \& D.J. Inman. Time-domain electromechanical impedance for structural health monitoring. In "8th International Conference on Structural Dynamics (Eurodyn 2011)" (2011), pp. 2043-2046.

[26] J. Vieira Filho, F.G. Baptista \& D.J. Inman. Time-domain analysis of piezoelectric impedance-based structural health monitoring using multilevel wavelet decomposition. Mechanical Systems and Signal Processing, 25(5) (2011), 1550-1558. 